\title{
A primary hepatic neuroendocrine tumor disguised as hepatocellular carcinoma
}

\author{
Zhihao Li, Chee-Chien Yong, Chao-Long Chen \\ Liver Transplantation Center, Department of Surgery, Kaohsiung Chang Gung Memorial Hospital, Kaohsiung \\ Correspondence to: Chao-Long Chen, MD, PhD (Hon). Liver Transplantation Center, Department of Surgery, Kaohsiung Chang Gung Memorial \\ Hospital, 123 Dapi Road, Niao-Sung, Kaohsiung. Email: clchen@cgmh.org.tw.
}

Submitted Dec 12, 2019. Accepted for publication Dec 23, 2019.

doi: $10.21037 /$ hbsn.2020.01.02

View this article at: http://dx.doi.org/10.21037/hbsn.2020.01.02

In a 72 -year-old female patient, a $2 \mathrm{~cm}$ large lesion over liver segment 7 (S7) was incidentally diagnosed during a hepatitis $\mathrm{C}$ follow-up. The patient was asymptomatic and had an unremarkable physical examination. Her past medical history consists of chronic hepatitis C, diabetes mellitus type 2 and arterial hypertension.

Her blood test results were normal except for highly elevated alpha-fetoprotein (AFP) $(821.32 \mathrm{ng} / \mathrm{mL})$. In the abdominal CT and MRI, the tumor lesion exhibited moderate arterial enhancement and early washout favoring the diagnosis of hepatocellular carcinoma (HCC) (Figure 1A,B,C,D). An HCC of cT1bN0M0 stage Ib was suspected and a partial S7 hepatectomy was performed. The resected tissue contained the tumor $(1.5 \mathrm{~cm} \times 1.4 \mathrm{~cm} \times 1.3 \mathrm{~cm})$ beneath the liver capsule with a $1.3 \mathrm{~cm}$ resection margin (Figure 1E).

Surprisingly, the pathology revealed a neuroendocrine tumor with small-sized neoplastic epithelial cells with hyperchromatic, pleomorphic nuclei and salt-pepperlike nuclear chromatin. The immunohistochemical characterization of the tumor was positive for synaptophysin and CD56. The Ki-67 index was $80 \%$, consistent with a grade 3 neuroendocrine carcinoma (NEC).

The patient had an uneventful postoperative recovery and underwent further studies to rule out an extrahepatic origin of the NEC: Both panendoscopy and colonoscopy were unremarkable. PET-CT studies performed with F-18FDG and Ga-68-DOTATOC isotopes did not detect any avid lesions (Figure 1F). Also, the AFP serum level returned to normal postoperatively. Hence the final diagnosis of primary hepatic neuroendocrine tumor (HPNET) was made.

The patient did not receive any adjuvant therapy because a R0 tumor resection was achieved without evidence of locoregional tumor progression. She was followed-up every 3 months with abdominal ultrasound and blood tests for the tumor markers AFP, CEA, CA19-9, which remained unremarkable.

PHNETs are extremely rare, with less than 150 cases reported in the English literature (1). They represent $0.3 \%$ of all NETs (2) which originate from neuro-ectodermal cells. Patients with PHNETs present in their 40-60 s with no gender preference (1). They remain asymptomatic for a long time due to slow tumor growth and lack of endocrine functionality. Unlike liver metastasis of NETs, only $6.8 \%$ of patients with PHNETs present with carcinoid syndrome (2). Hence PHNETs are either diagnosed incidentally or at a progressed stage due to tumor mass effect.

The rarity of PHNETs makes the differentiation from the more common HCCs difficult. One distinction between PHNETs and HCCs is that patients with HCCs typically have a history of alcoholic or viral hepatitis, liver cirrhosis, and elevated serum AFP levels, while patients with PHNETs have not (3). However, in congruence with Zhao's report (4), our patient with PHNET had a history of chronic hepatitis $\mathrm{C}$ and highly elevated serum AFP levels. Although the specificity of elevated AFP for HCC is high (80-94\%) (5), false-positive results can arise during pregnancy, active liver disease, embryonic or other gastrointestinal tumors. Since a pure AFP-producing NEC of the pancreas has been reported (6) and most PHNETs cases with elevated AFP levels were described in NECs, we hypothesize that NEC cells might re-express AFP in their dedifferentiation process.

The imaging modalities CT and MRI also have low 

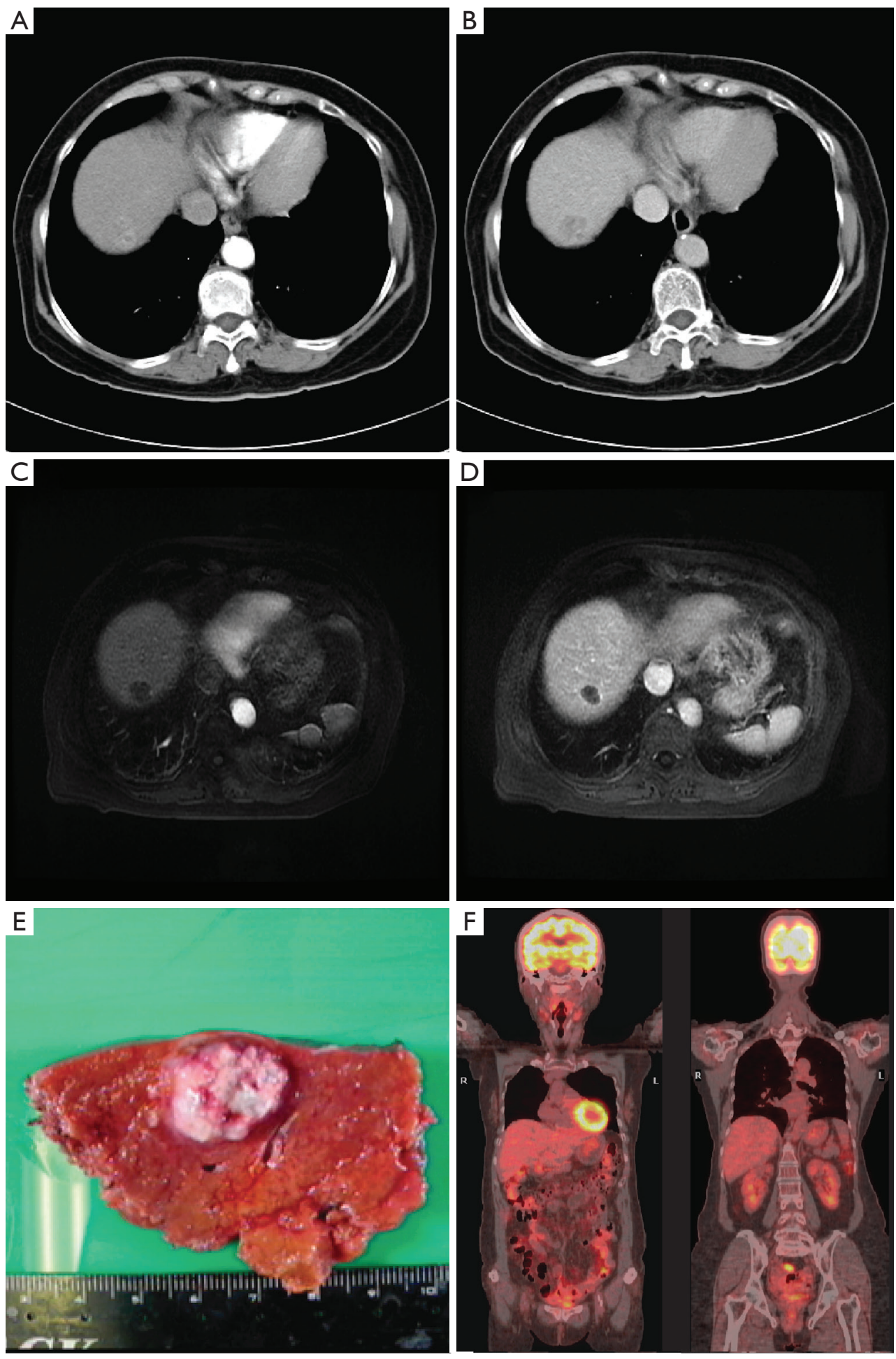

Figure 1 Radiologic and gross appearance of the PHNET. (A) Contrast-enhanced CT of the abdomen showing the $2 \mathrm{~cm}$ large focal hepatic lesion over S7 with moderate hyperenhancement in the arterial phase and (B) washout in the portal venous phase. (C) Contrast-enhanced MRI illustrating a S7 liver nodule $(2.4 \mathrm{~cm})$ hypoenhancement in the arterial phase and (D) early washout in the portal venous phase. (E) Crosssection of the resected liver tissue illustrating a $1.5 \mathrm{~cm}$ large solitary nodule capsulated by non-cirrhotic liver tissue. (F) PET-CT with F-18-FDG isotope performed after partial S7 hepatectomy, showing no pathologic uptake that would suggest another primary malignant lesion. 
specificity in differentiating PHNETs from HCCs. PHNETs have a rich blood supply from the hepatic artery and therefore exhibit hyperenhancement in the arterial phase and washout appearance in the portal venous phase of dynamic CT and MRI resembling HCCs. Both HCCs and PHNETs show a peripheral rim of smooth hyperenhancement in the portal venous or delayed phase pathologically correlating with a tumor capsule (7). In our case, the tumor lesion only exhibited moderate hyperenhancement in the arterial phase of the dynamic CT with hypoenhancing centers, suggesting tumor necrosis. It did not show the typical hyperenhancement in the arterial phase of dynamic MRI. These findings can be explained by the aggressiveness of the tumor with a Ki-67 of $80 \%$ outgrowing tumor angiogenesis causing tumor ischemia and necrosis. The resulting hypoperfusion leads to diminished contrast agent uptake and hence hypoenhancement in the dynamic CT and MRI.

Since the radiological and laboratory findings of PHNETs are not specific, definitive diagnosis of PHNETs needs pathologic evaluation of a surgically resected specimen. It does not only establish the diagnosis but also the tumor grading based on the mitotic rate and the Ki67 proliferation index which is essential for the treatment and prognosis (8). NECs often show morphologic features of small cell carcinoma and typically have a poor prognosis. The median overall survival of G3 PHNETs is significantly worse than G1 and G2, 42 months vs. 69 and 67 months respectively (8). There is no guideline for the treatment of PHNETs, however, complete surgical tumor resection is considered the only treatment of choice that can provide cure. Up to $85 \%$ of the tumors are resectable with surgery yielding a 5 -year survival rate of $74-78 \%$ and recurrence rate of $18 \%$ after hepatectomy (9). The outcome of PHNETs patients seems far better than the ones of HCCs because PHNETs patients are normally noncirrhotic while HCCs patients are. The outcomes become comparable when the 5-year survival and recurrence rate of non-cirrhotic HCC patients, $81 \%$ and $15 \%$ respectively, are taken as reference (10). Our patient with PHNEC has enjoyed a recurrence free survival of 4 years so far and reached the reported median survival of 42 months (8).

PHNETs are a diagnosis of exclusion since it is far less common than hepatic metastasis of NETs hence an extrahepatic primary NET must always be excluded first through studies including endoscopy, CT, MRI, Somatostatin PET-CT and a close follow-up. In our case, we could not find another primary tumor and the patient remained disease-free in the follow-ups, legitimizing the diagnosis of a PHNET.

In summary, diagnosis of PHNETs is difficult due to their rarity and unspecific laboratory and imaging findings that can easily be confounded with the more common HCC. Although the definitive diagnosis is only possible through pathologic confirmation and exclusion of an extrahepatic primary NET, we recommend taking the biochemical markers Chromogranin A, 5-Hydroxyindole Acetic Acid (5-HIAA), and Neuron Specific Enolase (NSE) whenever a NET is suspected. The standard of care is surgical tumor resection which yields good outcomes compared to other hepatic malignancy.

\section{Acknowledgments}

Funding: None.

\section{Footnote}

Provenance and Peer Review: This article was commissioned by the Editorial Office, Hepatobiliary Surgery and Nutrition. The article did not undergo external peer review.

Conflicts of Interest: All authors have completed the ICMJE uniform disclosure form (available at https://hbsn.amegroups. com/article/view/10.21037/hbsn.2020.01.02/coif). The authors have no conflicts of interest to declare.

Ethical Statement: The authors are accountable for all aspects of the work in ensuring that questions related to the accuracy or integrity of any part of the work are appropriately investigated and resolved. Written informed consent was obtained from the patient for publication of this manuscript and any accompanying images.

Open Access Statement: This is an Open Access article distributed in accordance with the Creative Commons Attribution-NonCommercial-NoDerivs 4.0 International License (CC BY-NC-ND 4.0), which permits the noncommercial replication and distribution of the article with the strict proviso that no changes or edits are made and the original work is properly cited (including links to both the formal publication through the relevant DOI and the license). See: https://creativecommons.org/licenses/by-nc$\mathrm{nd} / 4.0 \%$. 


\section{References}

1. Quartey B. Primary Hepatic Neuroendocrine Tumor: What Do We Know Now? World J Oncol 2011;2:209-16.

2. Modlin IM, Lye KD, Kidd M. A 5-decade analysis of 13,715 carcinoid tumors. Cancer 2003;97:934-59.

3. Chen Q, Chen X, Lu H, et al. Primary hepatic neuroendocrine tumors: retrospective analysis of seven cases and literature review. Transl Cancer Res 2018;7:428-40.

4. Zhao ZM, Wang J, Ugwuowo UC, et al. Primary hepatic neuroendocrine carcinoma: report of two cases and literature review. BMC Clin Pathol 2018;18:3.

5. Debruyne EN, Delanghe JR. Diagnosing and monitoring hepatocellular carcinoma with alpha-fetoprotein: new aspects and applications. Clin Chim Acta 2008;395:19-26.

6. Zhu X, Yong H, Zhang L, et al. Pure alpha-fetoprotein-

Cite this article as: $\mathrm{Li}$ Z, Yong CC, Chen CL. A primary hepatic neuroendocrine tumor disguised as hepatocellular carcinoma. HepatoBiliary Surg Nutr 2020;9(2):263-266. doi: 10.21037/hbsn.2020.01.02 producing neuroendocrine carcinoma of the pancreas: a case report. BMC Gastroenterol 2015;15:16.

7. Choi JY, Lee JM, Sirlin CB. CT and MR imaging diagnosis and staging of hepatocellular carcinoma: part II. Extracellular agents, hepatobiliary agents, and ancillary imaging features. Radiology 2014;273:30-50.

8. Shi C, Zhao Q, Dai B, et al. Primary hepatic neuroendocrine neoplasm: Long-time surgical outcome and prognosis. Medicine (Baltimore) 2018;97:e11764.

9. Knox CD, Anderson CD, Lamps LW, et al. Long-term survival after resection for primary hepatic carcinoid tumor. Ann Surg Oncol 2003;10:1171-5.

10. Taura K, Ikai I, Hatano E, et al. Influence of coexisting cirrhosis on outcomes after partial hepatic resection for hepatocellular carcinoma fulfilling the Milan criteria: an analysis of 293 patients. Surgery 2007;142:685-94. 